\title{
Intra-Tester Reliability and Concurrent Validity of Musculoskeletal Ultrasound and Vernier Calipre in Quantifying the Anteroposterior Diameter of the Common Extensor Origin of the Forearm and the Radial Nerve: A Cadaveric Study
}

Valentine Dones III

University of South Australia, donvc001@mymail.unisa.edu.au

Ian P. Aguinaldo

University of Santo Tomas

Svetlana Maris Aycardo

University of Santo Tomas

Buena Fe Apepe

University of Santo Tomas

Follow this and additional works at: https://nsuworks.nova.edu/ijahsp

Part of the Physical Therapy Commons, and the Physiotherapy Commons

\section{Recommended Citation}

Dones V, Aguinaldo IP, Aycardo SM, Apepe BF. Intra-Tester Reliability and Concurrent Validity of Musculoskeletal Ultrasound and Vernier Calipre in Quantifying the Anteroposterior Diameter of the Common Extensor Origin of the Forearm and the Radial Nerve: A Cadaveric Study. The Internet Journal of Allied Health Sciences and Practice. 2015 Jan 01;13(1), Article 9.

This Manuscript is brought to you for free and open access by the College of Health Care Sciences at NSUWorks. It has been accepted for inclusion in Internet Journal of Allied Health Sciences and Practice by an authorized editor of NSUWorks. For more information, please contact nsuworks@nova.edu. 


\title{
Intra-Tester Reliability and Concurrent Validity of Musculoskeletal Ultrasound and Vernier Calipre in Quantifying the Anteroposterior Diameter of the Common Extensor Origin of the Forearm and the Radial Nerve: A Cadaveric Study
}

\begin{abstract}
Introduction: The literature reports an increase in anteroposterior diameter of the common extensor origin (CEO) of the extensor carpi radialis brevis (ECRB) and extensor digitorum communis (EDC) or the radial nerve in painful elbows diagnosed with lateral epicondylalgia (LE) or supinator syndrome. The edge-to-edge measurements of these anatomical structures are quantified using musculoskeletal ultrasound (MSUS). However, in the current literature, reports on the reliability and validity of MSUS measurements of the CEO (of ECRB and EDC) and the radial nerve are not found. In this study, reliability was measured for three testers in determining the anteroposterior diameter of the CEO (of ECRB and EDC) and the radial nerve. Moreover, the concurrent validity was determined of MSUS measurements with Vernier calipre measurements using formalin preserved elbows of human cadavers. Methodology: Cadaver measurements of the CEO (of ECRB and EDC) and the radial nerve were performed. Initially, the sonologist measured the anteroposterior diameter of the CEO (of ECRB and EDC) and the radial nerve. A month after scan, the formalin preserved cadavers were dissected. Consequently, the anteroposterior diameter of the exposed CEO (of ECRB and EDC) and the radial nerve was measured using the Vernier calipre. Results: Eight upper extremities of four embalmed Filipino cadavers ( 2 males: 2 females) were dissected. A total of seven (7) CEO of EDC, seven (7) CEO of ECRB, and eight (8) radial nerves at the level of the radial head in the elbows of four (4) cadavers were measured using the MSUS and the Vernier calipre. The MSUS and Vernier calipre protocol used in this study was found to be reliable, $p>0.05$. However, in all three levels of interest, the MSUS measurements were statistically different from the Vernier calipre measurements, $p<0.05$. Conclusion: MSUS and Vernier calipre measurements are reliable methods in measuring the CEO (of ECRB and EDC) and the radial nerve. While each of these methods is reliable in measuring the anteroposterior dimensions of the CEO (of the EDC and ECRB) and the radial nerve, substituting one for the other yielded statistically different measurement results.
\end{abstract}

\section{Author Bio(s)}

- Valentin Dones III, PhD, Is a researcher with the International Centre for Allied Health Evidence at the University of South Australia in Adelaide, South Australia. He is also is an Assistant Professor, with the Centre for Health Research on Movement Sciences at the University of Santo Tomas, Manila.

- Ian P. Aguinaldo, MSPT, PTRP, is an Instructor with the College of Rehabilitation Sciences at the University of Santo Tomas, Manila.

- Svetlana Maris Aycardo, MD, is a Rehabilitation Specialist with the Faculty of Medicine and Surgery at the University of Santo Tomas, Manila.

- Buena Fe Apepe, MD is a Chair, Department of Anatomy with the Faculty of Medicine and Surgery at the University of Santo Tomas, Manila. 


\title{
IJAHSP \\ The Internet Joưnal of Allied Health Sciences and Practice \\ Dedicated to allied health professional practice and education
}

Vol. 13 No. 1 ISSN 1540-580X

\section{Intra-Tester Reliability and Concurrent Validity of Musculoskeletal Ultrasound and Vernier Calipre in Quantifying the Anteroposterior Diameter of the Common Extensor Origin of the Forearm and the Radial Nerve: A Cadaveric Study}

\author{
Valentin Dones III, $\mathrm{PhD}{ }^{1,2}$ \\ Ian P. Aguinaldo, MSPT, PTRP 3 \\ Svetlana Maris Aycardo, MD 4 \\ Buena Fe Apepe, MD 5
}

1. Researcher, International Centre for Allied Health Evidence, University of South Australia, South Australia

2. Assistant Professor, Centre for Health Research on Movement Sciences, University of Santo Tomas, Manila

3. Instructor, College of Rehabilitation Sciences, University of Santo Tomas, Manila

4. Rehabilitation Specialist, Faculty of Medicine and Surgery, University of Santo Tomas, Manila

5. Chairman, Department of Anatomy, Faculty of Medicine and Surgery, University of Santo Tomas, Manila

Australia

Philippines

CITATION: Dones V, Aguinaldo I, Aycardo S, Fe Apepe B. Intra-Tester Reliability and Concurrent Validity of Musculoskeletal Ultrasound and Vernier Calipre in Quantifying the Anteroposterior Diameter of the Common Extensor Origin of the Forearm and the Radial Nerve: A Cadaveric Study. The Internet Journal of Allied Health Sciences and Practice. Volume 13 Number 1.

\begin{abstract}
Introduction: The literature reports an increase in anteroposterior diameter of the common extensor origin (CEO) of the extensor carpi radialis brevis (ECRB) and extensor digitorum communis (EDC) or the radial nerve in painful elbows diagnosed with lateral epicondylalgia (LE) or supinator syndrome. The edge-to-edge measurements of these anatomical structures are quantified using musculoskeletal ultrasound (MSUS). However, in the current literature, reports on the reliability and validity of MSUS measurements of the CEO (of ECRB and EDC) and the radial nerve are not found. In this study, reliability was measured for three testers in determining the anteroposterior diameter of the CEO (of ECRB and EDC) and the radial nerve. Moreover, the concurrent validity was determined of MSUS measurements with Vernier calipre measurements using formalin preserved elbows of human cadavers. Methodology: Cadaver measurements of the CEO (of ECRB and EDC) and the radial nerve were performed. Initially, the sonologist measured the anteroposterior diameter of the CEO (of ECRB and EDC) and the radial nerve. A month after scan, the formalin preserved cadavers were dissected. Consequently, the anteroposterior diameter of the exposed CEO (of ECRB and EDC) and the radial nerve was measured using the Vernier calipre. Results: Eight upper extremities of four embalmed Filipino cadavers (2 males: 2 females) were dissected. A total of seven (7) CEO of EDC, seven (7) CEO of ECRB, and eight (8) radial nerves at the level of the radial head in the elbows of four (4) cadavers were measured using the MSUS and the Vernier calipre. The MSUS and Vernier calipre protocol used in this study was found to be reliable, $p>0.05$. However, in all three levels of interest, the MSUS measurements were statistically different from the Vernier calipre measurements, $p<0.05$. Conclusion: MSUS and Vernier calipre measurements are reliable methods in measuring the CEO (of ECRB and EDC) and the radial nerve. While each of these methods is reliable in measuring the anteroposterior dimensions of the CEO (of the EDC and ECRB) and the radial nerve, substituting one for the other yielded statistically different measurement results.
\end{abstract}

\section{INTRODUCTION}

Lateral epicondylalgia (LE) is a soft tissue injury manifesting as pain on the lateral aspect of the elbow. It is reported to be the most common cause of lateral elbow pain.1,2 The prevalence of LE ranges from 1 to $1.3 \%$ in men and from 1.1 to $4.0 \%$ in

(C) The Internet Journal of Allied Health Sciences and Practice, 2015 
women in the general population with no apparent gender bias.3

The clinical diagnosis of LE is based on the replication of lateral elbow pain of the patient by one of the Cozen's, Mill's or Maudsley's tests. ${ }^{4}$ These clinical provocation tests are believed to stress one or a combination of the extensor carpi radialis brevis (ECRB), extensor digitorum communis (EDC), and the radial nerve at the level of the radial head. ${ }^{5-8}$ In the Cozen test, resisted wrist extension compresses the radial nerve by the contracting ECRB. ${ }^{5}$ In the Mill test, passive wrist flexion increases the pressure in the elbow compressing the radial nerve and tensioning the CEO. ${ }^{6}$ In the Maudsley test, resisted third finger extension compresses the ECRB over the radial nerve ${ }^{7}$ or strains the EDC's slip to the middle finger near its attachment to the lateral epicondyle. ${ }^{8}$

At the level of the radial head, the two separable musculotendinous attachments of the EDC and ECRB form the common extensor origin (CEO) that attaches on the lateral epicondyle. ${ }^{9}$ The EDC is at CEO's superficial portion (approximately 65 to $75 \%$ of the CEO thickness) and the ECRB is at CEO's deepest quarter (approximately 25 to $35 \%$ of the CEO thickness). ${ }^{9}$ The fibrous edge of the ECRB angles downward and overlies the radial nerve. $5,7,10,11$

The CEO (of ECRB and EDC) and the radial nerve are strained during handgrip activities that use forceful and repetitive wrist extension and alternating forearm supination and pronation. ${ }^{3,12}$ As a response to the forceful and repetitive handgrip, seven studies using musculoskeletal ultrasound (MSUS) had reported echogenic abnormalities with three studies reporting on large anteroposterior diameters of the CEO (of ECRB and EDC) reflecting thickening in elbows with LE as reported in Table 1.13-19 Though not yet reported in elbows with LE, thickened anteroposterior diameter of the radial nerve has been reported in elbows with supinator syndrome.20

Table 1. Anteroposterior Diameter Measurements of CEO and RN by MSUS

\begin{tabular}{|c|c|c|c|c|c|c|}
\hline & \multicolumn{4}{|c|}{ Mean Anteroposterior Diameter Measurements of the CEO and the Radial Nerve (in mm) } \\
\hline & \multicolumn{2}{|c|}{ Toprak et al. $^{19}$} & \multicolumn{2}{c|}{ Lee et al. ${ }^{14}$} & \multicolumn{2}{c|}{ Bodner et al. ${ }^{20}$} \\
\hline CEO & Healthy elbows & $\begin{array}{c}\text { Affected } \\
\text { Elbows }\end{array}$ & $\begin{array}{c}\text { Healthy } \\
\text { elbows }\end{array}$ & $\begin{array}{c}\text { Affected } \\
\text { elbows }\end{array}$ & $\begin{array}{c}\text { Healthy } \\
\text { elbows }\end{array}$ & $\begin{array}{c}\text { Affected } \\
\text { elbows }\end{array}$ \\
\hline RN & 4.23 & 4.58 & $>4.2$ & $<4.2$ & NA & NA \\
\hline
\end{tabular}

Key: CEO, common extensor origin; mm, millimetre; MSUS, musculoskeletal ultrasound; NA, not applicable; RN, radial nerve

To quantify the anteroposterior diameter of the CEO (of ECRB and EDC) and the radial nerve in LE, there is an increase in the use of MSUS where edge-to-edge measurements of small, well-defined tendons and nerves are investigated. ${ }^{21-23}$ While MSUS can be useful in quantifying the anteroposterior diameter of the CEO (of ECRB and EDC) and the radial nerve, the results of MSUS are dependent on the skills of the operator and on the MSUS protocol used.24 Poltawski et al suggests that experts following the same protocols can have different quantitative measurements of same tendon/nerve and different tendons/nerves affecting the reliability of the measurement results. ${ }^{25}$ In the current literature, there is lack of reliable MSUS protocol to measure the anteroposterior diameter of the CEO (of ECRB and EDC) and the radial nerve.

In addition to the lack of a reliable MSUS protocol in measuring the anteroposterior diameter of the CEO (of ECRB and EDC) and the radial nerve, no concurrent validity study has compared the measurements of MSUS with actual measurements of Vernier calipre of the CEO (of ECRB and EDC) and the radial nerve in the human body. This is despite the availability of Vernier calipre that measures human soft tissues.26-29 Moreover, Vernier calipre measurements are used as the reference standard in validating the measurements obtained through MSUS. 26

Considering the gaps identified in the literature on the reliability and concurrent validity of MSUS and Vernier calipre in measuring the anteroposterior diameter of the CEO (of ECRB and EDC) and the radial nerve, this study aimed to determine the intra-tester reliability and concurrent validity of the sonologist in the use MSUS and the two testers in the use of the Vernier calipre in measuring the anteroposterior diameter of the following structures in the elbow at the level of the radial head: CEO of the ECRB, CEO of the EDC, and radial nerve.

\section{MATERIALS AND METHODS}

Ethics: This study was approved by the Ethics Review Board of the University of Santo Tomas (UST). 
Setting: The Human Anatomy Laboratory of the Faculty of Medicine and Surgery of the University of Santo Tomas.

Tester: For MSUS measurements of the CEO (of ECRB and EDC) and radial nerve, the rehabilitation doctor had 14 years of practice in rehabilitation medicine and had been using MSUS for the past five years. For Vernier calipre measurements of the CEO (of ECRB and EDC), the senior researcher was an Assistant Professor in Human Anatomy Laboratory 1 (Musculoskeletal System) and 2 (Organ System) with 11 years of experience in cadaver dissection. For Vernier calipre measurements of the radial nerve, the junior researcher has 3 years of experience in cadaver dissection.

Equipment: The ESaote MyLab 40 Family Ultrasound machine (ESaote Asia Pacific Diagnostic Private Limited) with a multifrequency broadband linear array transducer $(5$ to $17 \mathrm{MHz}$ ) was used to measure in millimeters $(\mathrm{mm})$ the anteroposterior diameter of the CEO (of the ECRB and EDC) and the radial nerve at the level of the radial head. A standard mechanical Vernier calipre accurate up to $0.05 \mathrm{~mm}$ was used to measure in millimeters $(\mathrm{mm})$ the structures of interest.

Study processes: As shown in Figure 1, the study processes include MSUS scan of non-dissected elbows by the sonologist, cadaver dissection by the senior researcher and Vernier calipre measurements by the senior and junior researchers.

Figure 1. Study Processes Involved In Determining the Measurements of the CEO (Of ECRB and EDC) and the Radial Nerve

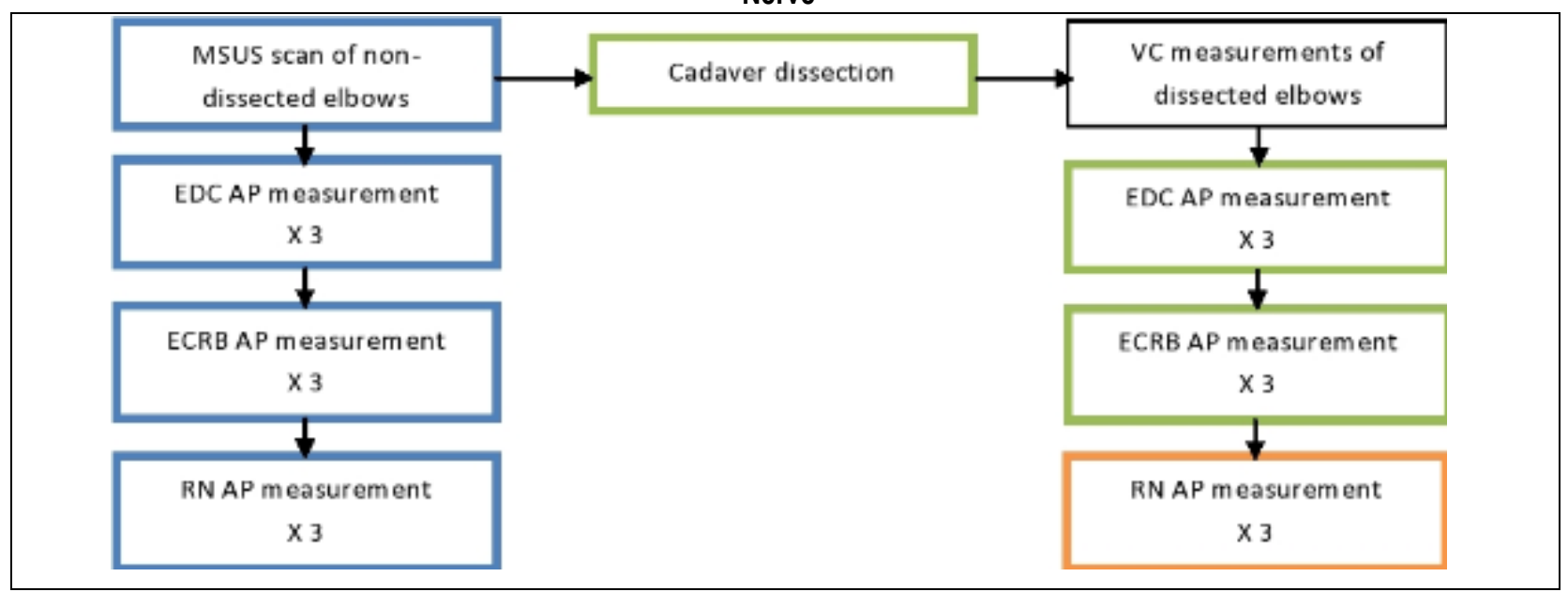

Keys: AP, anteroposterior; CEO, common extensor origin; ECRB, Extensor Carpi Radialis Brevis; EDC, Extenor Digitorum Communis; MSUS, musculoskeletal ultrasound; RN, radial nerve; VC, Vernier caliper. Blue boxes indicate sonlogist intervened processes. Green boxes indicate primary research-intercened processes. Orange box indicates junior research-intervened processes. Black box indicated senior and junior researchers-intervened processes.

MSUS scan of non-dissected elbows: Eight pronated non-dissected forearms of four cadavers held at 30 to 40 degrees of elbow flexion were scanned by the sonologist. Prior to scanning, the sonologist determined the radial head of the cadaver's elbow via palpation and transversely placed the transducer head immediately distal to the radial head. At the level immediately distal to the radial head, the anteroposterior diameter of the CEO (of ECRB and EDC) and the radial nerve was measured each for three (3) consecutive times as shown in Figure 1. The size of the ultrasound image was $407 \times 636$ pixels, with the image in 8-bit grayscale ( 8 bits per pixel with 256 shades of gray). A research assistant recorded the measurements.

Cadaver dissection: A month after the upper extremities were scanned by the sonologist, the senior researcher dissected the upper extremities. Using the sharp end of the scalpel, the skin and fascia overlying the lateral epicondyle and the area $5 \mathrm{~cm}$ distal to it were dissected away from the overlying EDC muscle. The exposed EDC muscle was cut $5 \mathrm{~cm}$ distal to the lateral epicondyle. To prevent fissuring of the EDC and ECRB muscle fibres, the blunt end of the scalpel was used to separate the EDC from the underlying ECRB.

Vernier calipre measurements of dissected elbows: At the level immediately distal to the radial head, the senior researcher measured the anteroposterior diameter of the tendon of the EDC and the tendon of the ECRB. After measuring the CEO (of the ECRB and EDC), the junior researcher measured the anteroposterior diameter of the radial nerve as shown in Figure 1. Each of the CEO (of ECRB and EDC) and the radial nerve was measured for three consecutive times. A research assistant recorded the measurements. 


\section{STATISTICAL ANALYSIS USED}

Medcalc for Windows, 2012-2013 was used to compute for the mean and 95\% confidence interval of the mean for each trial in the CEO of ECRB, the CEO of EDC, and the radial nerve. 30 Using Medcalc for Windows, 2003-2010, the Bland \& Altman plot (Bland \& Altman, 1986 and 1999) was used to:

a. Identify the statistical differences in the three trial measurements in each of the tendon of ECRB, the tendon of EDC, and the radial nerve. In each level of interest, trial 1 was compared against trials 2 and 3 . Additionally, trial 2 was compared against trial 3. In each level of interest, the mean of the trial measurements that were not statistically different was determined.

b. Identify the presence of systematic differences between mean scores of MSUS and Vernier calipre measurements in each level of interest. The differences between these two techniques were plotted against the averages of the MSUS and Vernier calipre measurements.

The line of equality was used to determine the presence of statistical differences in between trial measurements for each level of interest. Lines for $95 \%$ Confidence Interval $(\mathrm{Cl})$ of mean of differences defined the magnitude of statistical differences. Line of equality that is outside the $95 \% \mathrm{Cl}$ of mean of differences indicates presence of significant statistical differences in between trials. A p-value of less than 0.05 indicates presence of systematic differences between the three trial measurements for each level of interest and between the two techniques (MSUS and Vernier calipre measurements) used to measure the anatomical structures.

\section{RESULTS}

Eight upper extremities of four embalmed Filipino cadavers (2 males: 2 females) were dissected. No evidence of cuts and blotches indicating human-induced damage in the tissues and fractures of humerus, radius and ulna was observed.

A total of seven (7) CEO of EDC, seven (7) CEO of ECRB, and eight (8) radial nerves at the level of the radial head in the elbows of four (4) cadavers were measured using the MSUS and the Vernier calipre. The anteroposterior diameter of the CEO (of ECRB and EDC) in the elbow of one female cadaver was not measured due to advanced stage of decomposition.

Intra-tester reliability of the sonologist in measuring the CEO (of ECRB and EDC) and the radial nerve at the level of the radial head: A total of 66 MSUS measurements (21 CEO of the EDC, 21 CEO of the ECRB, and 24 radial nerve) were taken by the sonologist. In all three levels of interest, no statistical differences in between measurement trials were reported ( $p>0.05)$ as shown in Table 2.

Table 2. Mean ( $95 \% \mathrm{Cl}$ of the mean) of the CEO (of ECRB and EDC) and the radial nerve as measured by the sonologist using the MSUS

\begin{tabular}{|c|c|c|c|c|}
\hline Levels of Interest & $\begin{array}{c}\text { Trial 1 } \\
\text { Mean(95\% Cl) in mm }\end{array}$ & $\begin{array}{c}\text { Trial 2 } \\
\text { Mean }(\mathbf{9 5} \% \mathbf{C l}) \text { in } \mathbf{~ m m}\end{array}$ & $\begin{array}{c}\text { Trial 3 } \\
\text { Mean }(\mathbf{9 5 \%} \mathbf{~ C l}) \text { in } \mathbf{~ m m}\end{array}$ & p-value \\
\hline ECRB & $1.26(1.04-1.48)$ & $1.26(1.01-1.51)$ & $1.33(1.05-1.60)$ & $\mathrm{p}>0.05$ \\
\hline EDC & $1.03(0.67-1.39)$ & $1.10(0.90-1.31)$ & $1.20(0.94-1.5)$ & $\mathrm{p}>0.05$ \\
\hline Radial nerve & $0.95(0.81-1.09$ & $1.0(0.87-1.13)$ & $0.95(0.75-1.15)$ & $\mathrm{p}>0.05$ \\
\hline
\end{tabular}

Key: CEO, common extensor origin; $\mathrm{Cl}$, confidence interval; ECRB, extensor carpi radialis brevis; EDC, extensor digitorum communis; mm, millimetres; MSUS, musculoskeletal ultrasound

Intra-tester reliability of the senior researcher in measuring the CEO (of ECRB and EDC) at the level of the radial head: A total of 42 Vernier calipre measurements ( 21 tendon of EDC and 21 tendon of ECRB) were taken by the senior researcher. As shown in Table 3, no statistical differences in between the three trial measurements using the Vernier calipre for each of the two tendons were reported $(p>0.05)$. 
Table 3. Mean ( $95 \% \mathrm{Cl}$ of the Mean) of the CEO (Of ECRB and EDC) as Measured by the Senior Researcher Using the Vernier Calipre

\begin{tabular}{|c|c|c|c|c|}
\hline Levels of Interest & $\begin{array}{c}\text { Trial 1 } \\
\text { Mean }(\mathbf{9 5} \% \mathbf{~ C l}) \text { in } \mathbf{~ m m}\end{array}$ & $\begin{array}{c}\text { Trial 2 } \\
\text { Mean }(\mathbf{9 5 \%} \mathbf{~ C l ) ~ i n ~} \mathbf{~ m m}\end{array}$ & $\begin{array}{c}\text { Trial 3 } \\
\text { Mean } \mathbf{9 5 \%} \mathbf{~ C l}) \text { in } \mathbf{~ m m}\end{array}$ & p-value \\
\hline ECRB & $5.42(3.76-7.09)$ & $5.56(3.63-7.49)$ & $5.55(3.37-7.73)$ & $\mathrm{p}>0.05$ \\
\hline EDC & $6.21(3.92-8.50)$ & $6.22(4.36-8.09)$ & $6.60(4.63-8.56)$ & $\mathrm{p}>0.05$ \\
\hline
\end{tabular}

Key: CEO, common extensor origin; $\mathrm{Cl}$, confidence interval; $\mathrm{ECRB}$, extensor carpi radialis brevis; $\mathrm{EDC}$, extensor digitorum communis; mm, millimetres

Intra-tester reliability of the junior researcher in measuring the radial nerve at the level of the radial head: A total of 24 Vernier calipre measurements of the radial nerve were taken by the junior researcher. Except for trials 1 and 3 , no statistical differences in between trials 1 and 2 and in between trials 2 and 3 were reported $(p>0.05)$ as shown in Table 4. Figure 2 shows the Bland and Altman plot for trials 1 and 3 showing that the line of equality (orange line) was outside of the $95 \%$ confidence interval of the mean of differences (green line), indicating significant differences in between trials.

Table 4. Mean ( $95 \% \mathrm{Cl}$ of the Mean) of the Radial Nerve as Measured by the Junior Researcher Using the Vernier Calipre

\begin{tabular}{|c|c|c|c|c|}
\hline Levels of Interest & $\begin{array}{c}\text { Trial } 1 \\
\text { Mean }(95 \% \mathrm{Cl}) \text { in } \mathrm{mm}\end{array}$ & $\begin{array}{c}\text { Trial } 2 \\
\text { Mean }(95 \% \mathrm{Cl}) \text { in } \mathrm{mm}\end{array}$ & $\begin{array}{c}\text { Trial } 3 \\
\text { Mean }(95 \% \mathrm{Cl}) \text { in } \mathrm{mm}\end{array}$ & $p$-value \\
\hline Radial nerve & $2.03(1.38-2.68)$ & $1.80(1.11-2.49)$ & $1.73(1.02-2.43)$ & $\begin{array}{c}\mathrm{p}>0.05 \\
\text { except for } \\
\text { trials } 1 \text { and } 3\end{array}$ \\
\hline
\end{tabular}

Key: $\mathrm{Cl}$, confidence interval; $\mathrm{mm}$, millimetres

Figure 2. Bland Altman Plot of VC Measurements Between Trials 1 and 3

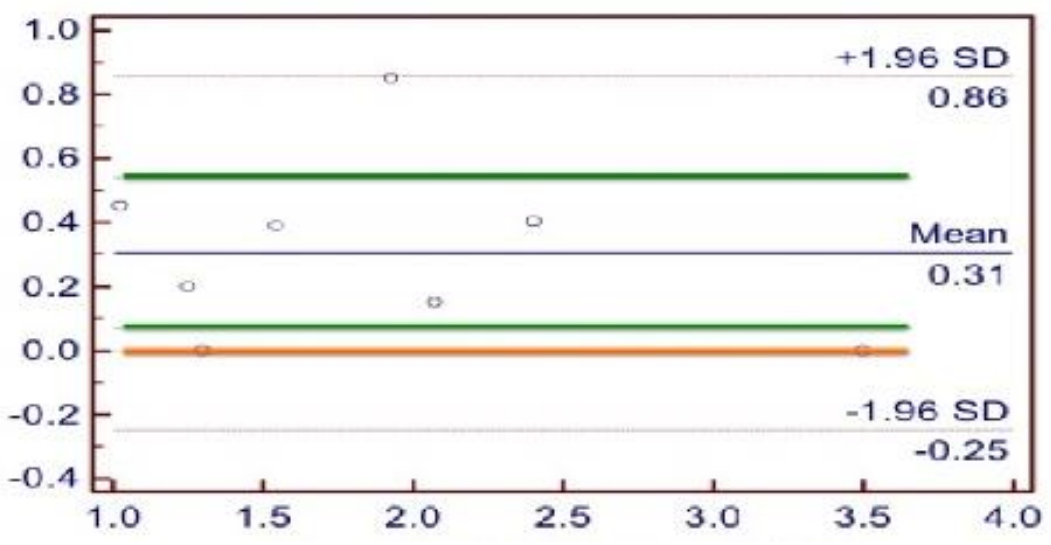

Key: VC, Vernier Calipre

Concurrent validity of MSUS and Vernier calipre in measuring the anteroposterior diameters of the CEO (of ECRB and EDC) and the radial nerve: The mean Vernier calipre measurements of the CEO (of ECRB and EDC) (average of three trials) and the mean Vernier calipre measurements of the radial nerve (average of trials 2 and 3) are significantly larger than their mean MSUS measurements (average of three trials) $(p<0.05)$ as shown in Table 5. The Bland and Altman plots for the CEO (of ECRB and EDC) and the radial nerve indicated statistical differences in between the MSUS and Vernier calipre measurements $(p<0.05)$.

Table 5. Mean MSUS and VC Measurements for the CEO of the ECRB and EDC and the Radial Nerve

\begin{tabular}{|c|c|c|c|}
\hline Levels of Interest & $\begin{array}{c}\text { MSUS Mean Measurements } \\
\text { Mean }(\mathbf{9 5} \% \mathbf{C I}) \text { in } \mathbf{~ m m}\end{array}$ & $\begin{array}{c}\text { VC Mean Measurements } \\
\text { Mean }(\mathbf{9 5} \% \mathbf{C l}) \text { in } \mathbf{~ m m}\end{array}$ & p-values \\
\hline ECRB & $1.26(1.03-1.48)$ & $5.51(3.62-7.41)$ & 0.002 \\
\hline EDC & $1.11(0.88-1.35)$ & $6.33(4.35-8.30)$ & 0.001 \\
\hline Radial nerve & $0.97(0.67-1.27)$ & $1.76(0.78-3.53)$ & 0.038 \\
\hline
\end{tabular}

Key: Cl, confidence interval; ECRB, extensor carpi radialis brevis; EDC, extensor digitorum communis; mm, millimetres; MSUS, musculoskeletal ultrasound; $\mathrm{VC}$, Vernier calipre 


\section{DISCUSSION}

This cadaver study investigated the intra-tester reliability and the concurrent validity of MSUS and Vernier calipre protocols in quantifying the anteroposterior diameter of the CEO (of ECRB and EDC) and the radial nerve. The study's findings were that a) The MSUS protocol used by the sonologist in measuring the anteroposterior diameter of the CEO (of ECRB and EDC) and the radial nerve was reliable; b) The Vernier calipre protocol used in measuring the anteroposterior diameter of the CEO (of ECRB and EDC) was more reliable compared to the Vernier calipre protocol used in measuring the anteroposterior diameter of the radial nerve; and c) In all three levels of interest, the MSUS measurements were statistically different from the Vernier calipre measurements.

The reliability of the sonologist in scanning the anteroposterior diameter of the CEO (of ECRB and EDC) and the radial nerve was underpinned by the standard MSUS protocol, the state-of-the art MSUS machine, and the expertise of the sonologist used in this study, confirming the factors influencing MSUS scan of anatomical structures reported in the paper of Poltawski et al. ${ }^{25}$ The MSUS protocol consists of palpation of the radial head prior to placement of the transducer head, standard positioning of the elbow during scan, and the use of copious gel. Determination of the level of the radial head by the sonologist standardised the placement of the transducer head on an area immediately distal to the radial head. This process improved the reliability of the sonologist, as it yielded MSUS images that showed approximately the same segment of the CEO (of ECRB and EDC) and the radial nerve across the eight non-dissected scanned elbows. To keep the MSUS image clear during actual measurement, the pronated forearm was kept at 30 to 40 degrees of flexion by the senior researcher. Moreover, the copious amount of gel kept the transducer head perpendicular and in maximal contact with the cadaver skin. Additionally, the sensitivity of the MSUS equipment set at automatic gain with depth of $2.7 \mathrm{~mm}$ ensured distinct edge-to-edge measurements of the CEO (of the ECRB and EDC) and the radial nerve. Considering that MSUS use is highly dependent on the skills of the operator, we are confident on the expertise of our sonologist who had 5 creditable years of scanning elbows of cadavers.

Of the three levels of interest, the statistical differences only seen in the Vernier calipre measurement trials 1 and 3 of the radial nerve could be secondary to the tight apposition of the radial nerve on the radial head. The convex radial head prevented the Vernier calipre clamp from being placed between the radial head and the radial nerve. To position the radial nerve in between the Vernier calipre clamps, the junior researcher lifted the radial nerve which could have altered the actual measurement of the anteroposterior diameter of the radial nerve.

The significantly statistical difference in anteroposterior diameters of the CEO (of the EDC and ECRB) and the radial nerve between MSUS and Vernier calipre measurements indicate that these two methods cannot be interchangeably used in determining the size of these anatomical structures. Considering that the Vernier calipre measurements were done one month after the MSUS measurements, we assumed that decomposition of cadaver tissues could have likely decreased the Vernier calipre measurements. However, this assumption does not support the larger Vernier calipre measurements of the CEO (of the ECRB and EDC) and the radial nerve compared to the MSUS measurements as shown in Table 4. Instead of decomposing soft tissue structures, we believe that the smaller MSUS measurements of the CEO (of ECRB and EDC) and the radial nerve in nondissected elbows was due to the pressure exerted by the intact yet formalin preserved and hardened skin and fascia on these anatomical structures consequently limiting their sizes. ${ }^{9}$ The pressure exerted on the radial nerve by the CEO (of the ECRB and EDC) limits its size, thus contributing to the smaller MSUS measurements compared to the Vernier calipre measurements. ${ }^{9,31}$ Comparing the measurements of the ECRB, EDC and radial nerve in this study with the measurements reported by Lee et al, Toprak et al, and Bodner et al, the Vernier calipre measurements of the three investigated anatomical structures in the formalin preserved cadavers closely approximate the MSUS measurements done on the elbows of healthy living participants as shown in Table 6.14,19,20 The difference in the MSUS measurements of our study with the MSUS measurements of Lee et al., Toprak et al., and Bodner et al., may be secondary to the process used in preserving the cadavers. ${ }^{14,19,20}$ The formalin in the tank where the cadavers were stored could have seeped into the skin and subcutaneous tissues potentially compressing the CEO (of ECRB and EDC) and the radial nerve, thus the resulting smaller MSUS measurements of these structures compared to the Vernier calipre measurements.

(C) The Internet Journal of Allied Health Sciences and Practice, 2015 
Table 6. Comparison of Anteroposterior Diameter Measurements of the CEO and the Radial Nerve

\begin{tabular}{|c|c|c|c|c|c|}
\hline & & \multicolumn{4}{|c|}{ Mean Anteroposterior Diameter Measurements Of The CEO And The Radial Nerve (In Mm) } \\
\hline \multirow{2}{*}{ MSUS } & & Current Study & Toprak et al. ${ }^{19}$ & Lee et al. ${ }^{14}$ & Bodner et al. ${ }^{20}$ \\
\cline { 2 - 6 } & ECRB & 1.26 & 4.23 & 4.22 & NA \\
\cline { 2 - 3 } & EDC & 1.11 & & & 1.33 \\
\cline { 2 - 3 } & RN & 0.97 & NA & NA & NA \\
\hline \multirow{2}{*}{ VC } & ECRB & 5.51 & NA & NA & \\
\cline { 2 - 3 } & EDC & 6.33 & & & \\
\cline { 2 - 3 } & RN & 1.76 & & & \\
\hline
\end{tabular}

Key: CEO, common extensor origin; ECRB, Extensor carpi radialis brevis; EDC, Extensor digitorum communis; mm, millimetre; $\mathrm{RN}$, radial nerve

\section{Limitations of the Study}

Considering that the cadavers were primarily intended for teaching undergraduate students of the College of Rehabilitation Sciences of the University of Santo Tomas, only eight upper extremities of four cadavers were included in this study. Decomposing human cadaver tissues could have affected (non-specifically) the measurement results as there was one month time lapse between MSUS and Vernier calipre measurements. Additionally, the intra-tester reliability of the testers could not be generalised to all sonologists and physiotherapists as the measurement process relies on multiple factors such as the expertise of the testers and equipment used for measuring sizes.

\section{Implications to Research and Practice}

Despite that the Vernier calipre is considered the reference standard in validating the measurements obtained through MSUS, factors such as the use of formalin preserved cadavers, decomposition of human cadaver tissues, and the design of the Vernier calipre used in this study potentially contributed to the disagreement in the obtained measurements between MSUS and Vernier calipre. Considering these factors, this study recommends the development of customised Vernier calipre probes for the measurement of radial nerve and use of fresh cadavers in validating MSUS measurements (with Vernier calipre measurements). Unless these factors are addressed in future research, the MSUS following a strict scan protocol is still practical and useful in performing edge-to-edge measurements of the CEO (of the ECRB and EDC) and the radial nerve. The changes in the size of these structures may characterise the severity of elbow condition as reported in the study of Lee et al, Toprak et al, and Bodner et al. $14,19,20$

\section{REFERENCES}

1. Kotnis NA, Chiavaras MM, Harish S. Lateral epicondylitis and beyond: imaging of lateral elbow pain with clinical-radiologic correlation. Skeletal Radiol. 2012;41:369-86. Doi: 10.1007/s00256-011-1343-8 [PMID: 22205505]

2. Faro F, Wolf J. Lateral epicondylitis: review and current concepts. J Hand Surg Am. 2007;32:1271-9. [PMID: 17923315]

3. Shiri R, Viikari-Juntura E. Lateral and medial epicondylitis: role of occupational factors. Clinical Rheumatol. 2011;25:43-57. Doi: 10.1016/j.berh.2011.01.013. [PMID: 21663849]

4. Lebrun C. What are the best diagnostic criteria for lateral epicondylitis? In: Wright JG. (ed) Evidence-based Orthopaedics: the Best Answers to Clinical Questions. 2008; London: Elsevier Health Sciences, 148-57.

5. Albrecht $\mathrm{S}$, Cordis $\mathrm{R}$, Kleihues $\mathrm{H}$, Noack W. Pathoanatomic findings in radiohumeral epicondylopathy. A combined anatomic and electromyographic study. Arch Orthop Traum Surg. 1997;116(3):157-63. [PMID: 9061171]

6. Erak S, Day R, Wang A. The role of supinator in the pathogenesis of chronic lateral elbow pain: a biomechanical study. $J$ Hand Surg-Brit Eur. 2004;29:461-4. [PMID: 15336750]

7. Roles NC, Maudsley RH. Radial tunnel syndrome: resistant tennis elbow as a nerve entrapment. J Bone Joint Surg-Brit Eur. 1972;54:499-508. [PMID: 4340924]

8. Fairbank SM, Corlett RJ. The role of the extensor digitorum communis muscle in lateral epicondylitis. J Hand Surg-Brit Eur. 2002;27:405-9. [PMID: 12367535]

9. Dones VCIII, Milanese S, Worth D, Grimmer-Somers K. The anatomy of the forearm extensor muscles and the fascia in the lateral aspect of the elbow joint Complex. Anat and Physiol: Curr Res. 2013;3:117. doi: 10.4172/2161-0940.1000117

10. Mazurek MT, Shin AY. Upper extremity peripheral nerve anatomy: current concepts and applications. Clin Orthop Relat $R$. 2001;383:7-20. [PMID: 11210971]

11. Yaxley G, Jull G. Adverse tension in the neural system. A preliminary study of the tennis elbow. Aus J Physiother. 1993;39:15-22. 
12. Haahr JP, Andersen JH. Physical and psychosocial risk factors for lateral epicondylitis: a population based case-referent study. Occup Environ Med. 2003;60:322-9. [PMID: 12709516]

13. Khoury V, Cardinal E. "Tenomalacia": a new sonographic sign of tendinopathy? Eur Radiol. 2009;19:144-6. Doi: 10.1007/s00330-008-1112-9 [PMID: 18677489]

14. Lee MH, Cha JG, Jin W, et al. Utility of sonographic measurement of the common tensor tendon in patients with lateral epicondylitis. Am J Roentgenol. 2011;196:1363-7. Doi: 10.2214/AJR/105769 [PMID: 21606300]

15. Maffulli N, Regine R, Carrillo F, Capasso G, Minelli S. Tennis elbow: an ultrasonographic study in tennis players. Brit J Sport Med. 1990;24:151-5. [PMID: 2078798]

16. Noh KH, Moon YL, Jacir AM, Kim KH, Gorthi V. Sonographic probe induced tenderness for lateral epicondylitis: an accurate technique to confirm the location of the lesion. KSSTA. 2010;18:836-9. Doi: 10.1007/s00167-009-1037-0 [PMID: 20111954]

17. Struijs PA, Spruyt M, Assendelft W J, Van Dijk CN. The predictive value of diagnostic sonography for the effectiveness of conservative treatment of tennis elbow. Am J Roentgenol. 2005;185:1113-8. [PMID: 16247118]

18. Tarhan S, Unlu S., Ovali Z, Pabuscu, Y. Value of ultrasonography on diagnosis and assessment of pain and grip strength in patients with Lateral Epicondylitis. Turk J Rheumatol. 2009;24:123-30.

19. Toprak U, Baskan B, Ustuner E, et al. Common extensor tendon thickness measurements at the radiocapitellar region in diagnosis of lateral elbow tendinopathy. Diagn Interv Radiol. 2012;18:566-70. Doi: 10.4261/1305-3825.DIR.5575-12.2 [PMID: 22498913]

20. Bodner G, Harpf C, Meirer R, Gardetto A, Kovacs P, Gruber H. Ultrasonographic appearance of supinator syndrome. J Ultras Med. 2002;21:1289-93. [PMID: 12418678]

21. Beekman R, Visser LH. High-resolution sonography of the peripheral nervous system -- a review of the literature. European J Neurol. 2004;11:305-14. [PMID: 15142223]

22. Walker FO, Cartwright MS., Wiesler ER, Caress J. Ultrasound of nerve and muscle. Clin Neurophysiol. 2004;115:495-507. [PMID: 15036045]

23. Heinmeyer $\mathrm{O}$, Reimers $\mathrm{CD}$. Ultrasound of radial, ulnar, median, and sciatic nerves in healthy subjects and patients with hereditary motor and sensory neuropathies. UMB. 1999;25:481-5. [PMID: 10374990]

24. Poltawski L, Jayaram V, Watson, T. Measurement issues in the sonographic assessment of tennis elbow. J Clin Ultrasound. 2010;38:196-204. Doi: 10.1002/jcu.20676 [PMID: 20186762]

25. Poltawski, L, Ali, S, Jayaram, V. Reliability of sonographic assessment of tendinopathy in tennis elbow. Skeletal Radiol. 2012;41:83-9. Doi: 10.1007/s00256-011-1132-4 [PMID: 21384199]

26. Sharma S, Thakur SL, Joshi SK, Kulkarni S. Measurement of gingival thickness using digital vernier caliper and ultrasonographic method: a comparative study. J Investig Clin Dent. 2013;4:1-6. doi: 10.1111/jicd.12026 [PMID: 23355379]

27. Borstad JD. Measurement of pectoralis minor muscle length: validation and clinical application. J Orthop Sport Phys Ther. 2008;38(4):169-74. doi:10.1016/j.semarthrit.2011.11.006 [PMID: 18434665]

28. Naderi S, Cakmakci H, Acar F, Arman C, Mertol T, Ardal MN. Anatomical and computed tomographic analysis of C1 vertebra. Clin Neurol Neurosur. 2003;105:245-8. doi:10.1016/S0303-8467(03)00037-4 [PMID: 12954539]

29. Schirmer UR, Wiltshire WA. Manual and computer-aided space analysis: A comparative study. Am J Orthod Dentofac. 1997;112(6):676-80. [PMID: 9423701]

30. MEDCALC for Windows statistics for biomedical research software manual, version 11.3, MedCalc easy-to-use statistical software, 2012-2013. Accessed 22 February 2013, http://www.medcalc.org/manual/index.php.

31. Mueller M, Maluf, K. Tissue adaptation to physical stress: a proposed "physical stress theory" to guide physical therapist practice, education, and research. Phys Ther. 2002;82:383-403. [PMID: 11922854] 